\title{
Studies on some economic and effective Ion exchange Resin used as catalyst in the synthesis of terpene derivative Isolongifoline Ketone
}

\author{
Vipin Kumar $^{1}$, Dr.A.K.Agarwal ${ }^{2}$ \\ Department of Chemistry, Bareilly College Bareilly, M.J.P.Rohilkhand University Bareilly (U.P.) 243005, India
}

\begin{abstract}
Terrenes are the abundant group of natural compounds that can be transformed into products of higher commercial value by organic reaction under the influence of suitable catalyst. Isolongifoline ketone was synthesized by Isolongifoline with the application of ion exchange catalyst viz. Tulsion T-421, Tulsion T-521, Indion 225, Indion 770.It is evident that Tulsion T-421 \& T-521 shows higher yield of Isolongifoline ketone due to its characteristics. Characterization of resin was done by determine the elemental analysis, ion exchange capacity, FTIR analysis, TGA and SEM analysis. The significance of the ion exchange resin is revealed by the conversion of Isolongifoline to Isolongifoline ketone.Thermax T-421was finding to possess the higher selectivity for isolongifoline ketone and high thermal stability.
\end{abstract}

Key words: Ion exchange Resin, Catalyst, Isolongifoline, Isolongifoline ketenes.

\section{Introduction}

Much effort is devoted to development of heterogeneous catalysis, their application in industrial-scale organic synthesis of fine chemicals and abatement of pollutants that are determined to environment ${ }^{\text {[1] }}$ Polystyrene supported sulfonic acid resins are widely used as acid catalysts in many kinds of petrochemical reactions, such as production of ethers ${ }^{[2,3,4]}$, alkylation. ${ }^{[5]}$, alkenes hydration ${ }^{[6]}$, esterification ${ }^{[7]}$ and other reactions. Strong Acid Resins are used in a number of instances as strong acid catalysts in place of soluble acids. The two main advantages of solid acid catalysis is the ease of separation of solid catalyst from the mixture and the fact that their selectivity and activity are often much better compared to that that of homogeneous acid catalyst. ${ }^{[8]}$ Most important resins of this type are formed from the copolymerization of styrene with divenylbenzene (DVB) as cross linking agent. The use of ion exchange resin catalysis has led to an improvement in the selectivity of the desired products. ${ }^{\text {[9] }}$ The acidity of resin is related to the number of hydrogen bridges forming an association of acid groups, which leads to a high sensitivity towards the amount of sorbed substrate or partial ion-exchange. In protophilic solvents the latter effects do not exist. ${ }^{[0]}$ Because of convenient access to diverse ranges of polymer supported structure and importance of acid catalyzed organic reactions in organic synthesis, these polymer-supported acid-catalyzed Reactions will continue to play critical roles in environmentally friendly organic synthesis and combinatorial library development. ${ }^{[11]}$

Several kinds of sulfonic acid catalysts based both on polymer and carbon materials were investigated .Due to the higher quality and more stability of attached $-\mathrm{SO} 3 \mathrm{H}$ groups on polymer based catalysts compared with carbon based catalysts, polymer based catalysts show better catalytic activity and recyclability. Moreover, introducing sulfonic acid $(-\mathrm{SO} 3 \mathrm{H})$ groups on organic-based materials is more desirable for enhancing the catalytic activity because the organic-based materials in general exhibit a hydrophobic feature. ${ }^{[12]}$

Sulfonated polystyrene catalysts show excellent performance in many chemical reactions, due to their high concentrations of acid sites. Extensive work has been carried out in synthesis of terpene derivatives by using ion exchange resin as catalyst. Production of isolongifoline from longifoline is carried out by using macro porous strong acid type cationic exchange resin as catalyst. ${ }^{[13]}$ Terpene- catechol was synthesized with turpentine oil and catechol as raw material, strong acidic ion exchange resins as catalyst. ${ }^{[14]}$

The object of the present study is the application of ion exchange catalyst (Tulsion T-421, Tulsion T521 , Indion 225, and Indion 770) in the oxidation of Isolongifolne to isolongifoline ketone \& Characterization of catalyst.

\subsection{Material}

\section{Material And Methods}

Commercial Ion Exchange Resin- Tulsion T-421H,Tulsion T-521H (Thermax),Indion 225, Indion 770, 421 (Ion Exchange),Isolongifoline (camphor and Allied products Bareilly), Hydrogen peroxide, Acetic acid (Qualigen) were used.Gellular Ion exchange bed is shown in Fig. 2.1. 


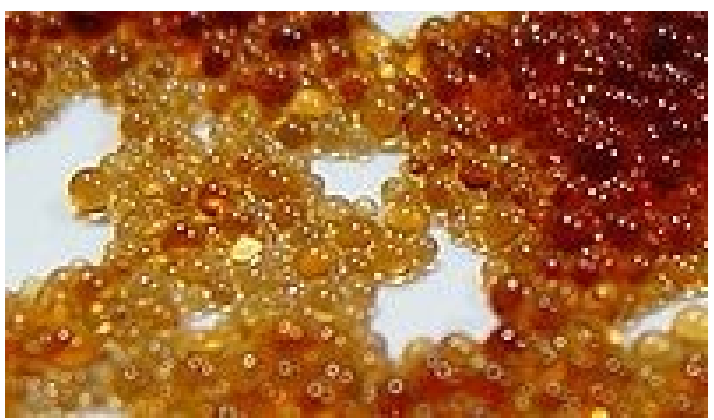

Fig.2.1. Gellular Ion exchange Resin bed

\subsection{Synthesis of Isolongifoline Ketone}

In a 1 lit. Three- necked round-bottom flask, equipped with a thermometer pocket and dropping funnel and mechanical stirrer. Water bath is used for heating purpose and temperature is maintained by thermostat regulator. Round-bottom flask is charged with $204 \mathrm{gm}$ (1mole) Iso-Longifoline, $180 \mathrm{gm}$ (3moles) Acetic Acid and $10 \mathrm{gm}$ catalyst resin. Start the heating and temperature of reaction mixture is maintained at $30-35{ }^{\circ} \mathrm{C}$, Dropping funnel is charged with $68 \mathrm{gm}$ (1mole) of $50 \%$ Hydrogen peroxide is added drop wise to solution with continuously stirring up to $1 \mathrm{hrs}$ and temp is maintained below $50^{\circ} \mathrm{C}$ during addition of hydrogen peroxide by thermostat regulator. The reaction mixture is stirred throughout the entire course of the reaction and the reaction temperature was slowly raised and maintained at $55-60^{\circ} \mathrm{C}$ up to $5 \mathrm{hrs}$. Reaction mixture is transferred to beaker by decanting process and catalyst is remained in the reaction flask. Three more experiments were repeated with remaining catalyst mass while keeping the same mole ratio of other inputs to check the catalytic performance with maximum use in $5 \mathrm{hrs}$ reaction cycles. Four experiments are performed by each catalyst (Tulsion T-421H, Tulsion T-521H, Indion 225, Indion 770) catalyst in the same mole ratio and reaction condition to check the performance of individual catalyst.

\subsection{Analysis of Reaction Mixture}

An aliquot of the reaction mixture is withdrawn and washed with sodium bicarbonate solution and analyzed using gas chromatograph-Nucon 5700 with chromatographic condition-Column:FFPEAT1000,Length:1.5meter. Initial temperature: $140{ }^{\circ} \mathrm{C}$, Final Temperature: $230{ }^{0} \mathrm{C}$, Rate: $4{ }^{0} \mathrm{C} @$ minute, Detector- Flame Ionization Detector (FID), Temperature: $250{ }^{\circ} \mathrm{C}$, Inj. Port Temperature: $230^{\circ} \mathrm{C}$, Carrier Gas: Nitrogen, Rate- $30 \mathrm{ml} / \mathrm{min}$, and Solvent: Acetone.The performance of catalyst is shown in Results and Discussion.

\subsection{Characterization of the Resin}

Characterization was done by following methods.

\subsubsection{Elemental analysis (CHS) and Ion Exchange capacity}

Catalyst elemental analysis refers to CHS analysis - the determination of the percentage weights of carbon, hydrogen, and heteroatom's (sulphur) of a sample. This information is important to help ascertain the structure and purity of a compound. The elemental analysis data was collected on an Elemental Analyzer Elementer Vario EL III Cario Erba 1108, over a wide range of sample metrics and concentrations with is this instrument.

Ion Exchange capacity is measured by Titration. A sample of resin is pretreated with $0.2 \mathrm{M} \mathrm{NaOH}$ and allowed to stand for 12 hrs and a known volume of supernatant is titrated with $0.05 \mathrm{M} \mathrm{H} 2 \mathrm{So} 4$ solution by using methyl orange indicator. This provides the strong acid capacity. This capacity is assumed equivalent to the total capacity for Cation resin with sulphonic acid functional groups.

\subsubsection{Thermo gravimetric analysis (TGA)}

Thermo gravimetric Analysis (TGA) were performed on Perkin Elmer,dimond TGA thermal analysis system between $40^{\circ} \mathrm{C}-730^{\circ} \mathrm{C}$. The measurement of resin samples were carried out in gas flow $(85 \mathrm{ml} / \mathrm{min})$ and heating rate $10^{\circ} \mathrm{C} / \mathrm{min}$. The mass of resin sample used was $1-10 \mathrm{mg}$.

\subsubsection{Scanning electron microscopy (SEM)}

Scanning electron microscopy has been applied to the surface studies for both topography a, from the sample surface, is studied upon exposure to a beam of high energy electrons .In the samples SEM images were measured on JEOL Model JSM-6390LV with EDS make JEOL Model JED-2300 with an acceleration voltage of $20 \mathrm{kV}$ and magnification $40,500,5000$ in vacuum \& image mode SEI. 


\subsection{Synthesis of Isolongifoline ketone}

\section{Results And Discussion}

The catalytic performance of catalyst - Thermax T-421H, Thermax T-521, Indion 225, and Indion 770 was evaluated by using epoxidation reaction hydrogen peroxide and acetic acid with Isolongifoline (purity 87.01\%). Table 3.1 and Fig.3.1 represent the catalytic performance of different Catalyst All catalyst shows the stable recyclability up to $4^{\text {th }}$ reaction as per experiments conducted in same mole ratio and reaction condition. The Conversion of Isolongifoline by using Indion 770 is very less as compared to other catalyst however conversion of Isolongifoline by Thermax T-421 H is higher with others. The results of Isolongifoline are consistent with results of $\mathrm{S}$ content.

Table 3.1: Catalytic conversion of Isolongifoline over Different Catalysts

\begin{tabular}{|l|l|l|l|l|}
\hline \multirow{2}{*}{ Catalyst } & \multicolumn{4}{|l|}{ Selectivity of Isolongifoline Ketone in Reaction Cycles (Reaction Time 5 Hrs) } \\
\cline { 2 - 5 } & $\mathbf{1}$ & $\mathbf{2}$ & $\mathbf{3}$ & $\mathbf{4}$ \\
\hline Thermax T-421 H & 90.05 & 89.44 & 84.55 & 81.45 \\
\hline Thermax T-521H & 83.42 & 82.57 & 81.96 & 78.19 \\
\hline Indion 225 & 78.43 & 77.16 & 75.0 & 71.18 \\
\hline Indion 770 & 61.41 & 58.84 & 57.6 & 54.03 \\
\hline
\end{tabular}

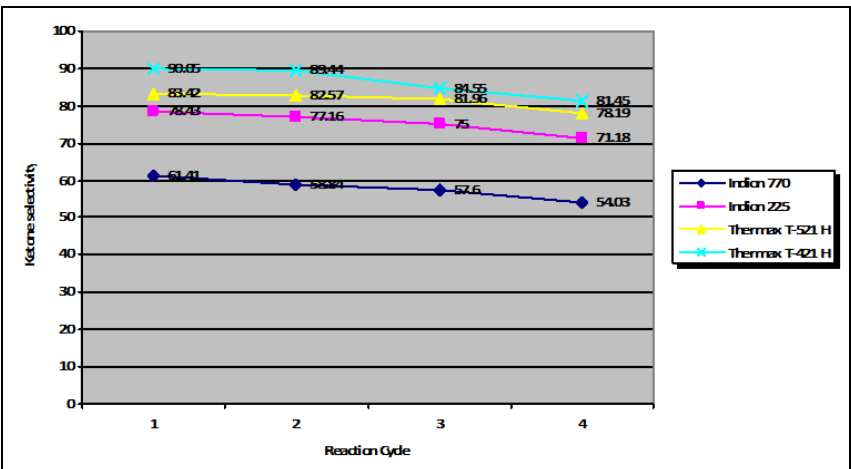

Fig. 3.1 Selectivity of ILF Ketone over Different Catalyst

\subsection{Characterization of catalysts}

\subsubsection{Elemental analysis (CHS) and Ion Exchange capacity}

The elemental compositions of the catalysts are listed in Table 3.2. High S content indicates the higher Ion exchange capacity that is large amount of attached - $\mathrm{SO} 3 \mathrm{H}$ group in polymer catalysts. It can be assumed that the by adding of divinylbenzene and increase of sulfonation temperature can facilitate the attachment of $\mathrm{SO} 3 \mathrm{H}$ groups. The acid amount determined by titration showed the same trend as the result of elemental analysis.

Table 3.2 Compositions of samples analyzed using elemental analysis

\begin{tabular}{|l|l|l|l|l|}
\hline Sample & $\mathrm{C}($ wt \%) & $\mathrm{H}(\mathrm{wt} \%)$ & $\mathrm{S}($ wt \%) & Ion exchange capacity $(\mathrm{meq} / \mathrm{ml})$ \\
\hline Thermax T-421 H & 34.61 & 5.75 & 10.64 & 1.84 \\
\hline Thermax T-521H & 38.86 & 5.68 & 11.27 & 1.96 \\
\hline Indion 225 & 34.57 & 5.59 & 10.58 & 1.80 \\
\hline Indion 770 & 31.61 & 5.02 & 9.74 & 1.46 \\
\hline
\end{tabular}

\subsubsection{Thermo gravimetric analysis (TGA)}

TG curves (Figure 3.2 to Figure 3.5) represents a dynamic weight loss profile of resin Thermax T-421, Thermax T-521, Indion 225 and Indion 770 respectively. All sulfonated polymer spheres show the loss in weight in separate temperature ranges. The weight loss up to $200^{\circ} \mathrm{C}$ can be attributed to moisture content. The weight loss from $200^{\circ} \mathrm{C}$ to $400{ }^{\circ} \mathrm{C}$ can be ascribed to the decomposition of -SO3H groups which is caused by the decomposition of - $\mathrm{SO} 3 \mathrm{H}$ groups (Table 3.3) by assuming the decomposition reaction produces $\mathrm{SO}$. The observed weight loss values in temp range $200{ }^{\circ} \mathrm{C}$ to $400{ }^{\circ} \mathrm{C}$ is evaluated for all catalyst. The observed weight loss for Thermax $\mathrm{T}-421 \mathrm{H}$ is lower than that of other resin, which indicates that the thermal stability of attached-SO3H groups of cross-linked sulfonated polymer in Thermax $\mathrm{T}-421 \mathrm{H}$ is more than other resin. The observed weight loss value for Catalyst $\mathrm{T}-521 \mathrm{H}$ is higher than others, which indicate the more $\mathrm{S}$ content in Thermax-T-

521H.The observed weight loss values for Indion 225 and Indion 770 is higher than Thermax T-421H, which might be due to main polymer chain degradation occurred along with the decomposition of - $\mathrm{SO} 3 \mathrm{H}$ groups or suggests that not all attached $-\mathrm{SO} 3 \mathrm{H}$ groups have been released when the polymer main chain begins 
to degrade. It is assumed that the thermal stability of attached -SO3H groups for cross-linked sulfonated polymer catalysts increased by the adding of divinylbenzene with increase of sulfonation temperature.

Table 3.3 Decomposition temperatures of samples.

\begin{tabular}{|c|c|c|c|c|}
\hline Sample & $\begin{array}{l}\text { Weight change }(\%) \\
\text { attributed to moisture } \\
\text { content } \\
\text { (weight loss up to } 200^{\circ} \mathrm{C} \\
\text { ) }\end{array}$ & $\begin{array}{l}\text { Weight change (\%) caused } \\
\text { by decomposition of - } \\
\mathrm{SO}_{3} \mathrm{H} \text { group } \\
\text { (weight loss from } 200^{\circ} \mathrm{C} \text { to } \\
400^{\circ} \mathrm{C} \text { ) }\end{array}$ & $\begin{array}{l}\text { Weight change caused } \\
\text { by degradation of } \\
\text {-main polymer chain } \\
\text { (weight loss from } 400^{\circ} \mathrm{C} \text { to } \\
730^{\circ} \mathrm{C} \text { ) }\end{array}$ & $\begin{array}{l}\text { Weight }(\%) \text { of un- } \\
\text { degradation of main } \\
\text { polymer chain after } \\
730^{\circ} \mathrm{C}\end{array}$ \\
\hline Thermax T-421 H & 28.33 & 31.41 & 14.45 & 25.81 \\
\hline Thermax T-521 H & 20.44 & 42.85 & 26.73 & 9.98 \\
\hline Indion 770 & 24.59 & 35.57 & 17.61 & 22.23 \\
\hline
\end{tabular}

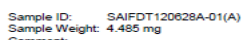

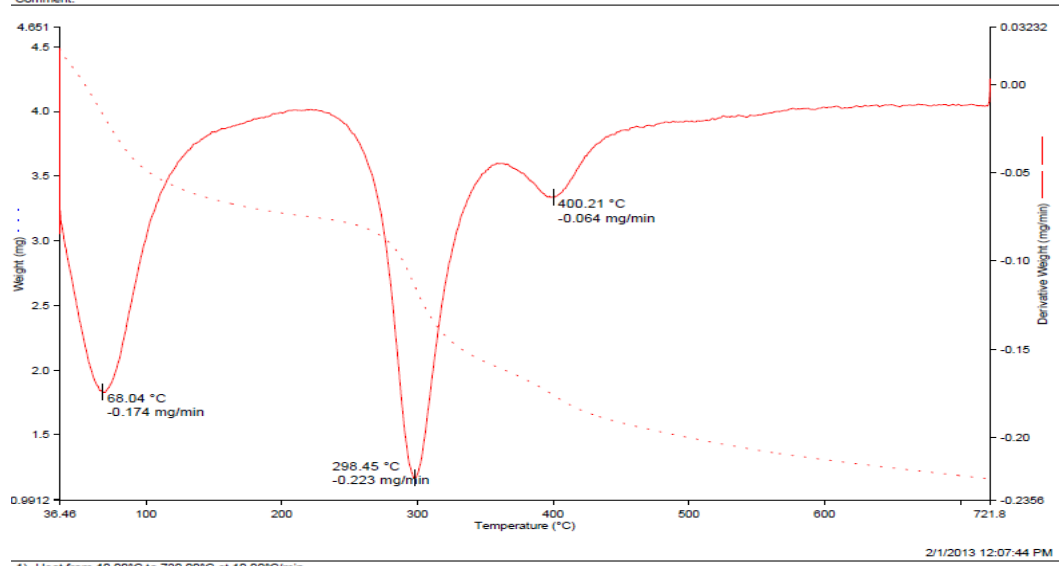

Fig. 3.2 TGA graph of Thermax T-421 H

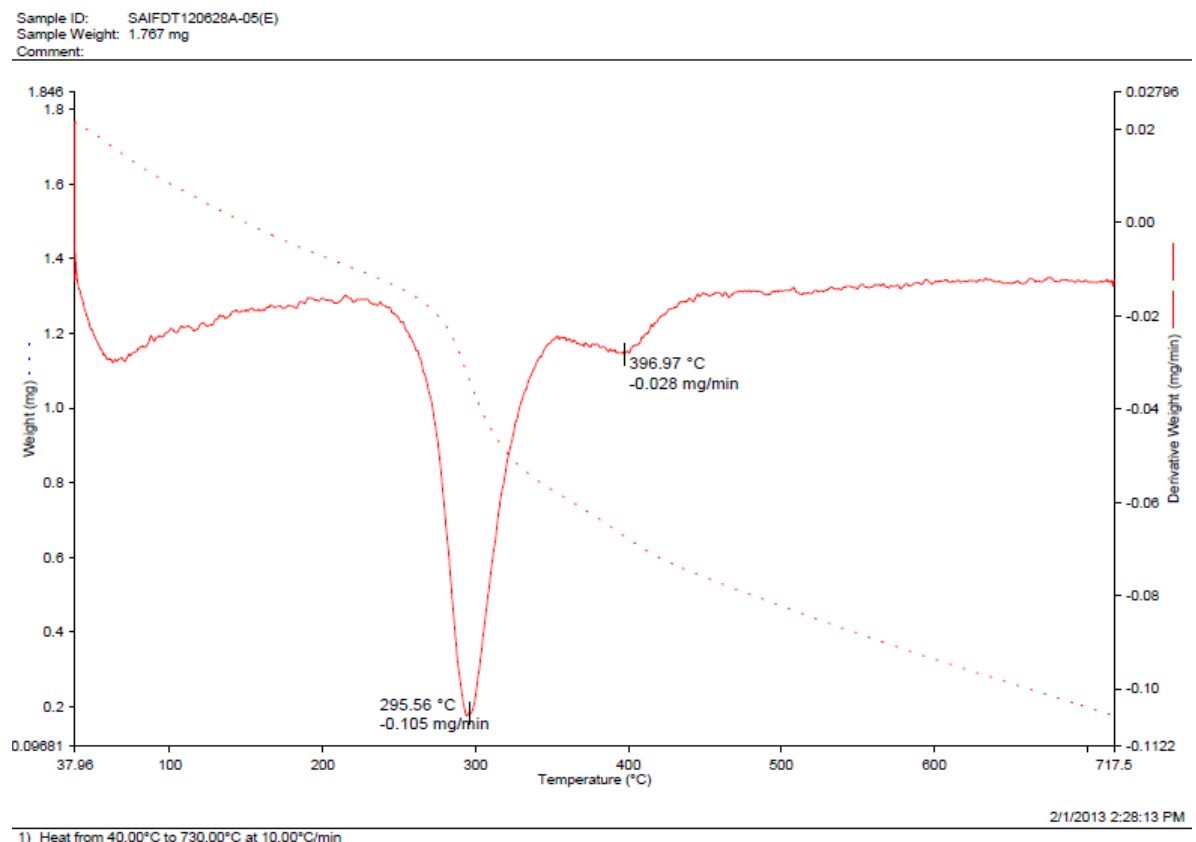

Fig. 3.3 TGA graph of Thermax 521H 


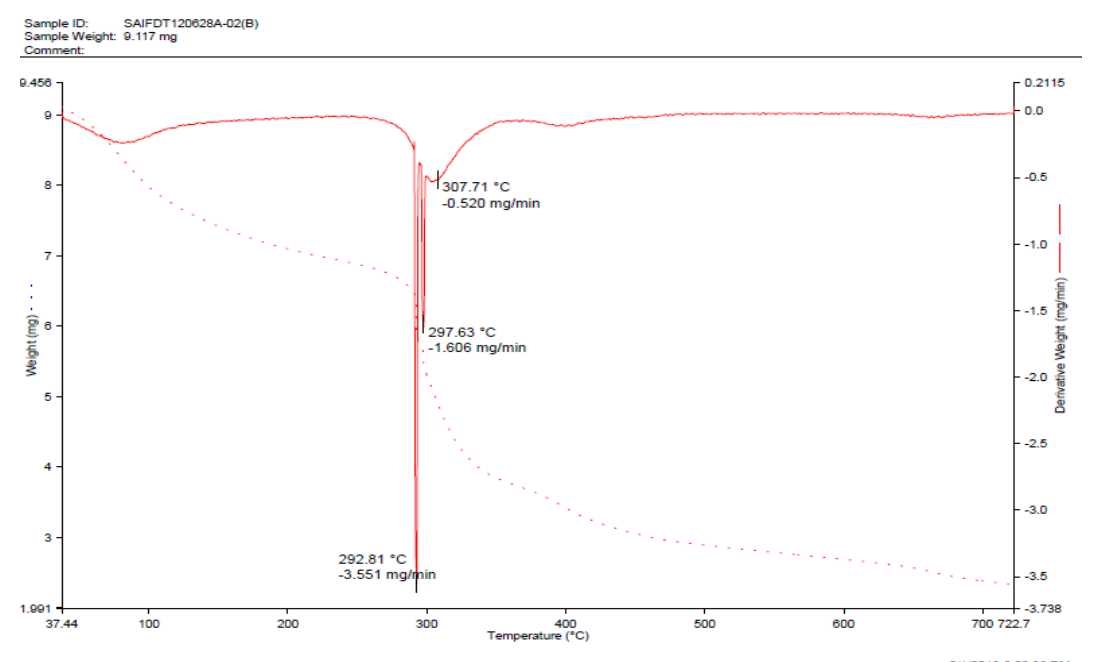

Fig. 3.4 TGA graph of Indion 225

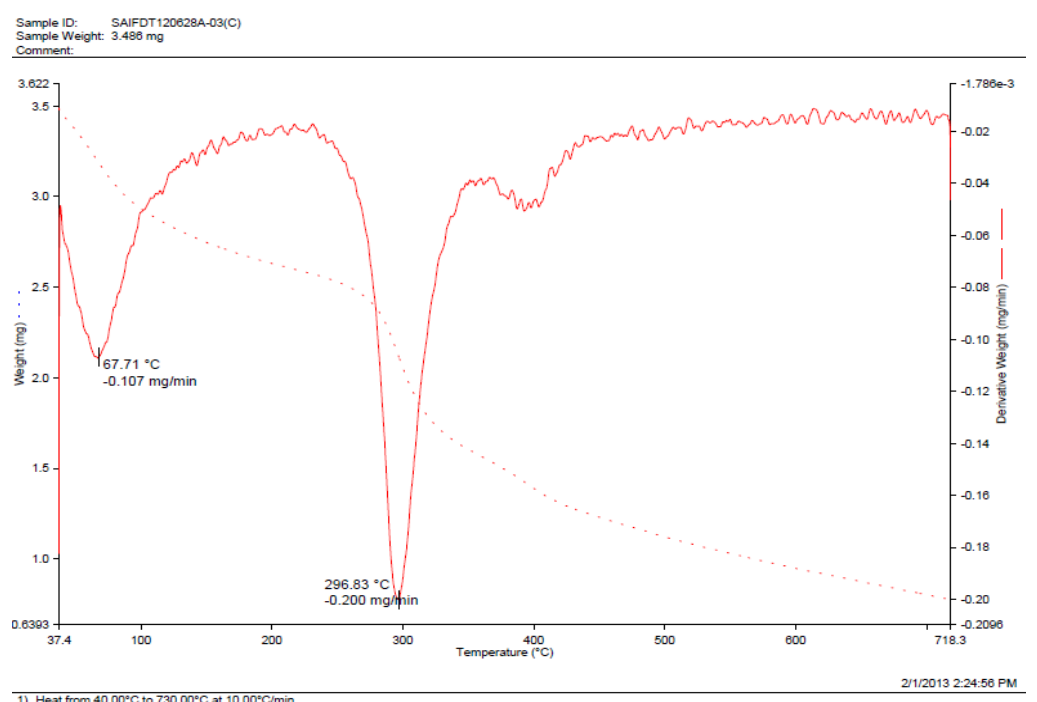

Fig. 3.5 TGA graph of Indion 770

\subsubsection{Scanning Electron Microscopy Analysis}

Fig. 3.6, 3.8, $3.10 \& 3.12$ shows the FESEM images of catalyst Thermax T-421H, Thermax T-521H, Indion 225, Indion 770 respectively. Fig. shows the diameter of polymer sphere of different resin. It can be seen that the diameter of polymer spheres are not uniform in all FESEM image and diameter of polymer sphere of Thermax $\mathrm{T}-421 \mathrm{H}$ is much uniform and less in dia as compared to other polymer sphere. It is assumed that diameter of resultant polymer spheres decreased with the increase of divinylbenzene during sulphonation process. Because the adding of DVB will cause the Varity of cross-link density for resultant spheres, therefore the more tightly cross-linked spheres show the smaller diameter. Divinylbenzene is more reactive than styrene, which will deduce the variety of cross-link density for the resultant crosslink polymer spheres. It is possible that the cross-linked polystyrene-divinylbenzene spheres have tightly cross-linked cores, however, more lightly cross-linked or linear-linked polystyrene chains are attached, which causes a core-shell structure ${ }^{[15]}$ The choice of particle size is a compromise; fine resins deliver a higher capacity .Coarse beads on the other hand more sensitive to osmotic stress and have slower kinetics, thus giving a lowering ion exchange capacity. Fig. 3.10 (FSEM image of Indion 225) is indicating large cracks in some polymer sphere beds, the numbers of cracked beads is important part of beads quality, cracking will lowers the -stability of resin. Fig. 3.7,3.9,3.11,3.13 shows the FESEM surface images at magnification x5000 of catalyst Thermax T-421H,Thermax T$521 \mathrm{H}$,Indion 225, Indion 770 respectively, It can be seen that the surface morphology of Resin Indion 225 \& Indion 770 is indicating rough surface while the morphology of Thermax T-421H is indicating plane spherical structure. In Fig. 3.7 (FSEM image of surface of Resin Thermax T-421H) we can see that the transparency of the outside of spheres is higher than that of the core of the spheres of other resin, which indicates the different density. It is also confirmed by the results of TGA (Table 3.3) which indicate that the thermal stability of attached- - $\mathrm{SO} 3 \mathrm{H}$ groups of cross-linked sulfonated polymer in Thermax T-421H is more than other resin. It can 
be concluded that little amount of divinylbenzene improve the stability of polymer during the sulfonation reaction process and increase the number of attached - $\mathrm{SO} 3 \mathrm{H}$ groups. While with the continuous adding of divinylbenzene the number of attached - $\mathrm{SO} 3 \mathrm{H}$ groups decreased due to the tighten core-shell structure

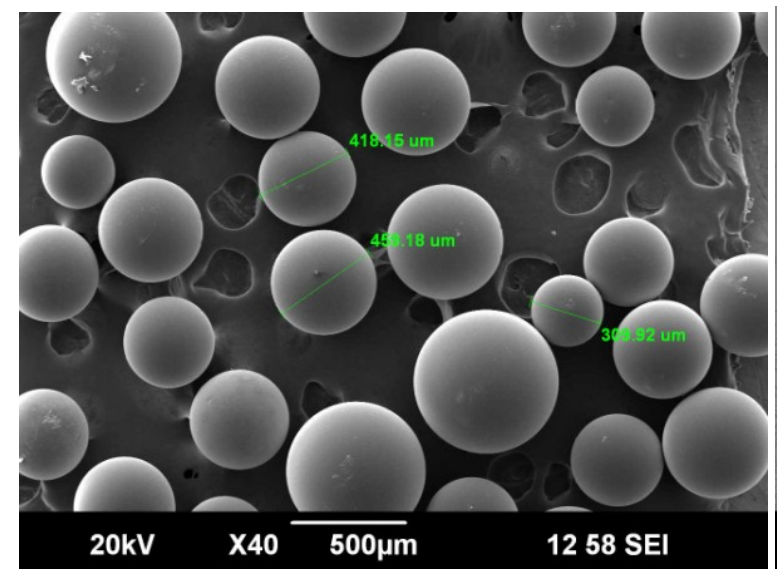

Fig.3.6 FSEM image (Topography) of Catalyst Thermax T-421H

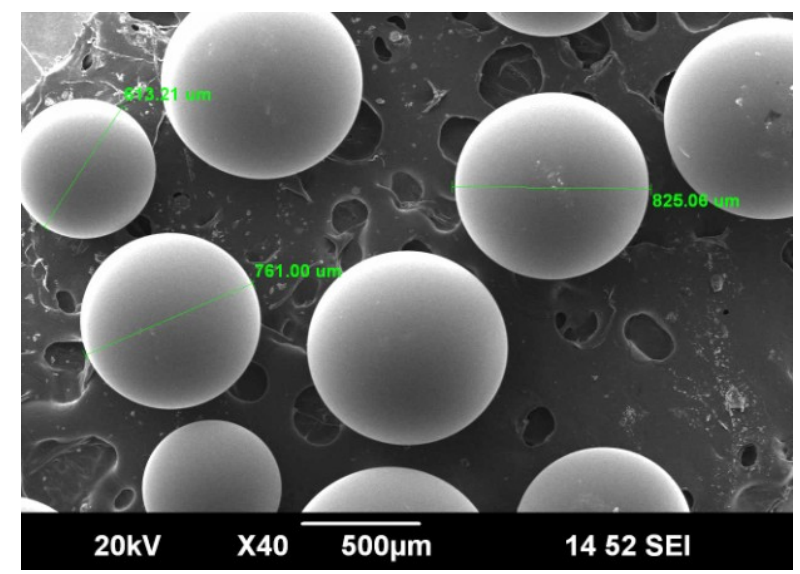

Fig. 3.8 FSEM image (Topography) of Catalyst Thermax T-521H

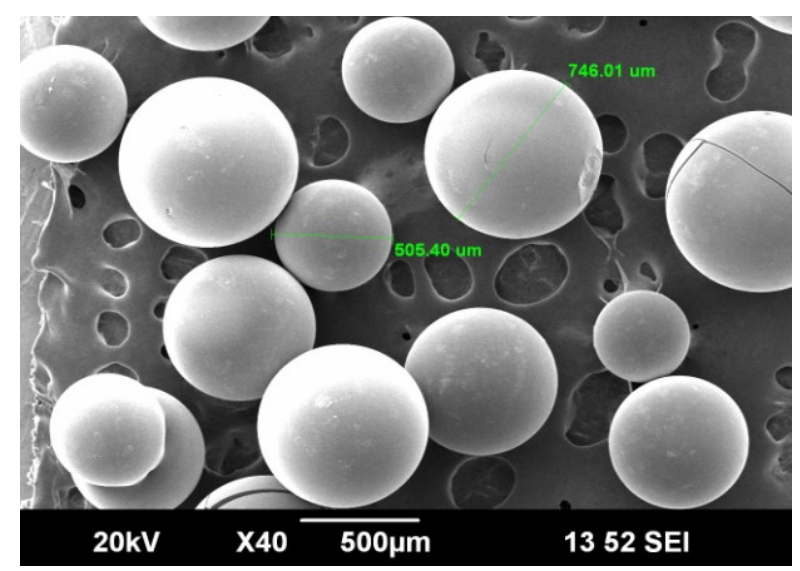

Fig. 3.10 FSEM image (Topography) of Catalyst Indion 225

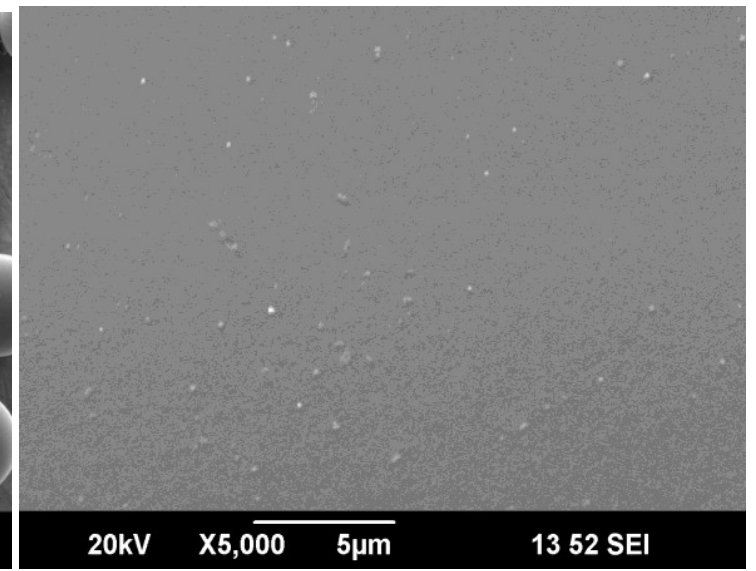

Fig.3.7 FSEM image of the surface of the Thermax $\mathrm{T}-421 \mathrm{H}$ at magnification $\mathrm{x} 5000$

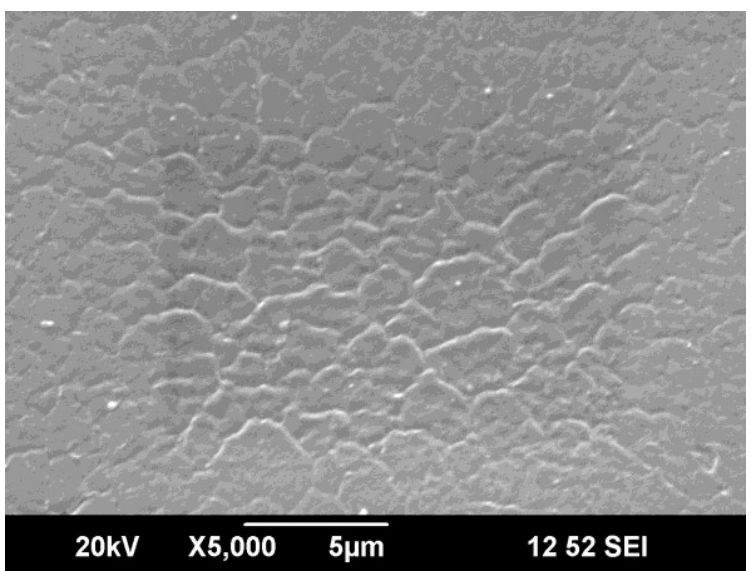

Fig.3.9 FSEM image of the surface of the Thermax $\mathrm{T}-521 \mathrm{H}$ at magnification $\mathrm{x} 5000$

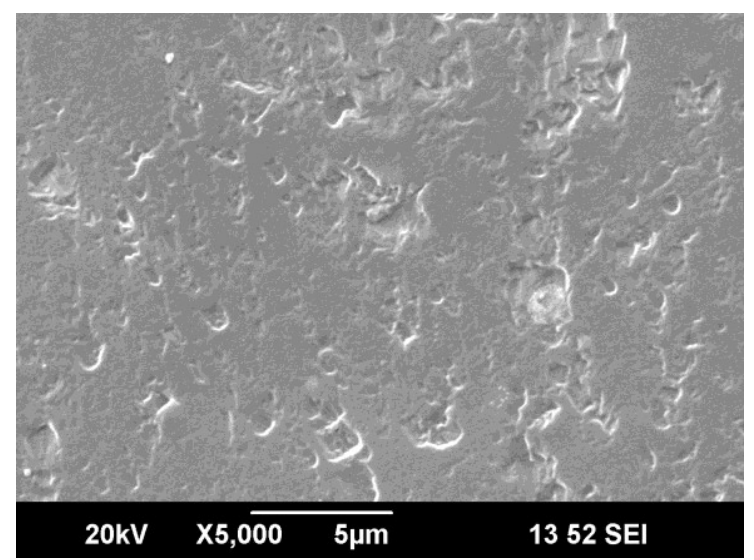

Fig.3.11 FSEM image of the surface of the Thermax Indion 225 at magnification $\times 5000$ 


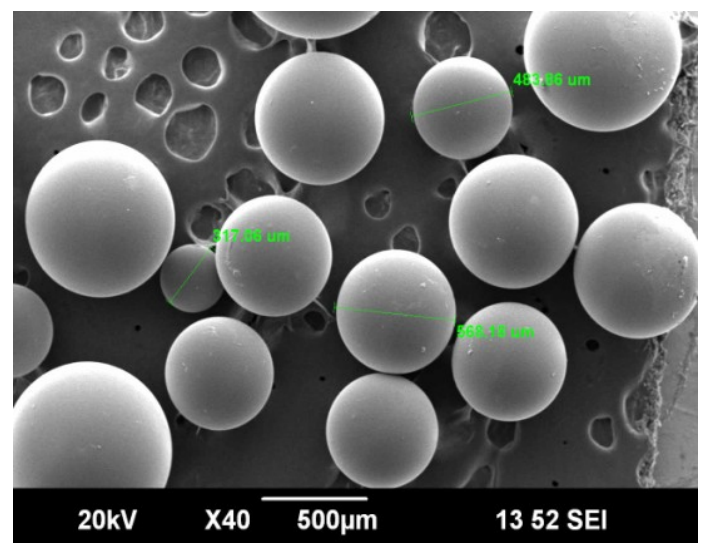

Fig. 3.12 FSEM image (Topography) of Catalyst Indion 770

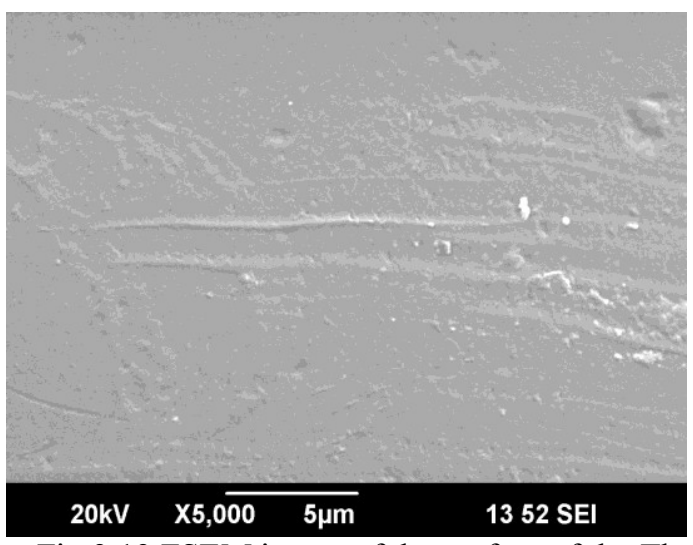

Fig.3.13 FSEM image of the surface of the Thermax Indion 770 at magnification $\mathrm{x} 5000$

\section{Conclusion}

In this work Isolongifoline ketone have been synthesized by using different sulphonic acid catalyst (Resin) and recyclability of resin in epoxidation reaction were evaluateted.The characterization of resins were carried out by method of elemental analysis, Ion exchange capacity, FTIR, TGA and SEM analysis. It was found that amount and stability of -So3H groups, particle size, and surface structure and cross linkage in polymer for resin plays a role in catalyst performance. The catalyst Thermax T-421H and Thermax T-521 shows better selectivity of Isolongifoline Ketone while Indion 770 shows lowest selectivity of Isolongifoline ketone, The conclusion that have been drawn that surface morphology of Resin Indion 225 \& Indion 770 is rough surface while the surface morphology of Thermax T- $421 \mathrm{H}$ is plane spherical structure and transparency of the outside of spheres is higher than that of the core of the spheres of other resin, which indicates the different density. Which is also confirmed by the results of TGA (Table 3.3) which indicate that the thermal stability of attached- - $\mathrm{SO} 3 \mathrm{H}$ groups of cross-linked sulfonated polymer in Thermax T- $421 \mathrm{H}$ is more than other resin hence Catalyst Thermax $\mathrm{T}-421 \mathrm{H}$ shows highest selectivity of Isolongifoline ketone.

\section{Acknowledgements}

The Authors are thankful to Dr. R.B.Singh, Head of the department of chemistry and Dr. R.P.Singh, Principal, Bareilly College Bareilly for providing facility to carry out research work. The Authors also thank STIC, Cochin University, Cochin for TGA and SEM analysis and STIC Lucknow for elemental analysis.

\section{References}

[1] Baiker A.;" Heterogeneous catalysis, From Fundamentals to Reactions Engineering Chimmia, 50, 1996, .65-73.

[2] Rehfinger, A. and U.Hoffmann. Kinetics of Methyl Tertiary Butyl Ether Liquid-phase Synthesis Catalyzed by Ion-Exchange Resin Macropor diffusion of Methanol as Rate Controlling Step.Eng.Sci. 45, 1990, 1619-1626.

[3] Iimura, S., K. Manabe and S. Kobayashi, Hydrophobic, low-loading and alkylated Polystyrene-supported sulfonic acid for Several organic reactions in water: remarkable Effects of both the polymer structures and loading levels of sulphuric Acids. Org. Chem., 1, 2003, 2416-2418.

[4] Boz, N., T. Dogu, K. Murtezaoglu and G. Dogu, Effect of hydrogen ion-exchange Capacity on activity of resin catalysts in tertamyl-Ethyl-ether synthesis. Appl. Catal. A-Gen., 268, 2004, 175-182.

[5] Sharma, M. M. Some novel aspects of cationic ion-exchange resins as catalysts. React. Funct. Polym. 26, $1995,3-23$.

[6] Hart, M., G. Fuller, D. R. Brown, C. Park, M. A. Keane, J. A. Dale, C. M. Fougret and R. W. Cockman. Acidities and catalytic activities of persulfonated poly (styrene- codivinylbenzene) Ion-exchange resins.Catal. Lett. 72, 2001, 135-139.

[7] Sanz, M. T., R. Murga, S. Beltran, J. L. Sanz, M. T., R. Murga, S. Beltran, J. L. Cabezas and J. Coca. Auto catalyzed and Ion exchange-resin-catalyzed esterification Kinetics of lactic acid with methanol. Ind Eng. Chem. Res., 41, 2002, 512-517.

[8] Yadav, G. D. and R. D. Bhagat. Experimental and theoretical analysis of Friedel-Crafts Acylation of thioanisole to 4-(methylthio) Acetophenone using solid acids. J. Mol. Catal. A-Chem., 235, 2005, 98-107.

[9] Chakrabarti, A. and Sharma, M.M. Cationic exchange resins as Catalysis Reactive Polymers 20 (1-2), 1993, 1-45.

[10] Christoph Buttersack "Accessibility and catalytic activity of sulfonic acid ion-exchange Resins solvents"- Research Institute of the Sugar Industry, Technical University, 3300 Braunschweig in different, F.R.G., Available online 17 April, 2003.

[11] Christoph Buttersack "Accessibility Chang, Ying; Bae, Chulsung, Polymer-Supported Acid Catalysis in Organic Synthesis: Source: Volume 8 (29), April, 2011, 208-236.

[12] Iimura, S., K. Manabe and S. Kobayashi, Hydrophobic polymer-supported catalyst for Organic reactions in water: Acid-catalyzed hydrolysis of thioesters and transprotection of thiols. Org. Lett., 5, 2003, 101-103.

[13] Zhang Zhibing, Zhou Zheng, Liu Hongjun, Liang Yinchun: Method and apparatus for preparing isolongifoline from longifoline, International classification: CO7c13/605, C07C5/22, Applicant- Nanjing Univ, Publication- 10.12.2005.

[14] Huang Dao-Zhan, LEI Fu-hou, LAN HongyunWU: Study on Synthesis of Terpene-Catechol with cation Exchanger Resin as Catalyst (Department of chemistry and Chemical Engineering, Guangxi Univ. for Nationalities, Nanning 30006 (China) Journals of Chinese --03-001, 2001

[15] Ding, Z.Y., S.M.Ma, D.Kriz, J.J.Aklonis and R Salovey...Model filled polymers.9. Synthesis of uniformly cross-linked polystyrene microbeads...Polym.Sci.Pt.B-polym.Phy. 30, 1992, 1189-1194. 